\title{
NeW Metric FOR HWMP PRotocol (NMH)
}

\author{
Sidi Ould Cheikh, Malik Mubashir Hassan and Abdelhak Geuroui \\ PRiSM Laboratory, University of Versailles, Versailles, France \\ sidi.ould-cheikh@prism.uvsq.fr, malik.hassaneprism.uvsq.fr, \\ moguedprism.uvsq. fr
}

\begin{abstract}
The IEEE 802.11s Wireless Mesh Networks (WMN) is a new multi-hop technology increasing the coverage of IEEE 802.11 Wireless Network and providing Internet access. Recently, many researchers proposed several metric routing protocols for wireless mesh networks (WMNs). Some metrics consider the interferences and other study the channel diversity of the distant links along the path between the source and the destination. In order to provide the best path between the source and the destination, this paper proposes a new routing metric named (NMH) New Metric for Hybrid Wireless Mesh Protocol(HWMP). $\mathrm{NMH}$ is based on two hop channel diversity and hop delay. The simulation results show that NMH outperforms WCETT in terms of average network throughput, end-to-end delay and number of flows.
\end{abstract}

\section{KEYWORDS}

Wireless Mesh Networks, Hybrid wireless mesh protocol, Routing metric, $802.11 \mathrm{~s}$

\section{INTRODUCTION}

The IEEE 802.11 Standard [1] provides Wireless Local Area Networks (WLAN) with one or two hop topologies and defines two modes of deployment. The first mode is composed by an Access Point (AP) and a number of associated stations. The second mode is an ad hoc configuration formed only by stations. The IEEE 802.11s Wireless Mesh Network (WMN) [2] is characterized by multi-hop topology, simplicity of deployment and providing Internet connectivity by low cost. The client level is composed of static or mobile stations and the Mesh Basic Service Set (MBSS) or Mesh level is formed by statics Mesh Points. A Mesh Point (MP) supports forwarding, path selection at layer-2 and multi-hop communications. In addition to MP functionalities, Mesh Access Points (MAP) have an AP function and Mesh Portal Point (MPP) bridge or interconnect the WMN with a non-802.11 network (Figure.1 illustrates this concept). Additionally, unicast, multicast and broadcast communications are supported by WMN. Three main approaches, that differ to implement mesh functionality, that differ in the protocol layer implementation (MAC, network and in-between layer). The MAC (layer-2) approach provides frames forwarding and path selection at layer-2, while the in-between layer (layer-2.5) approach uses an additional software layer interposed between layer-2 and layer-3.

The layer-3 approach implements mesh functionality at network layer [3]. The WMN based-IEEE 802.11s task group, proposed a layer-2 approach, MAC layer routing protocol HWMP (Hybrid Wireless Mesh Protocol), based on MAC addresses [2].

The performance is significantly degraded if a node have only single interface. In order to improve the performance, the node can use multiple wireless interfaces [15].

DOI : $10.5121 /$ ijcnc.2013.5204 
Existing routing metrics usually select paths that require the minimum transmission time for a packet in a path. However, a high-throughput path is one that can deliver a packet with the shortest end-to-end delay[16]. To provide the better path between the source and the destination, we propose a new metric based on two hop channel diversity combined with packet hop delay. This new routing metric named NMH is used by Hybrid Wireless Mesh Protocol(HWMP).

The rest of paper is organized as follows. Section 2 presents the related work, section 3 describes the pro-posed metric NMH and section 4 presents simulation results. Finally section 5 concludes the paper.

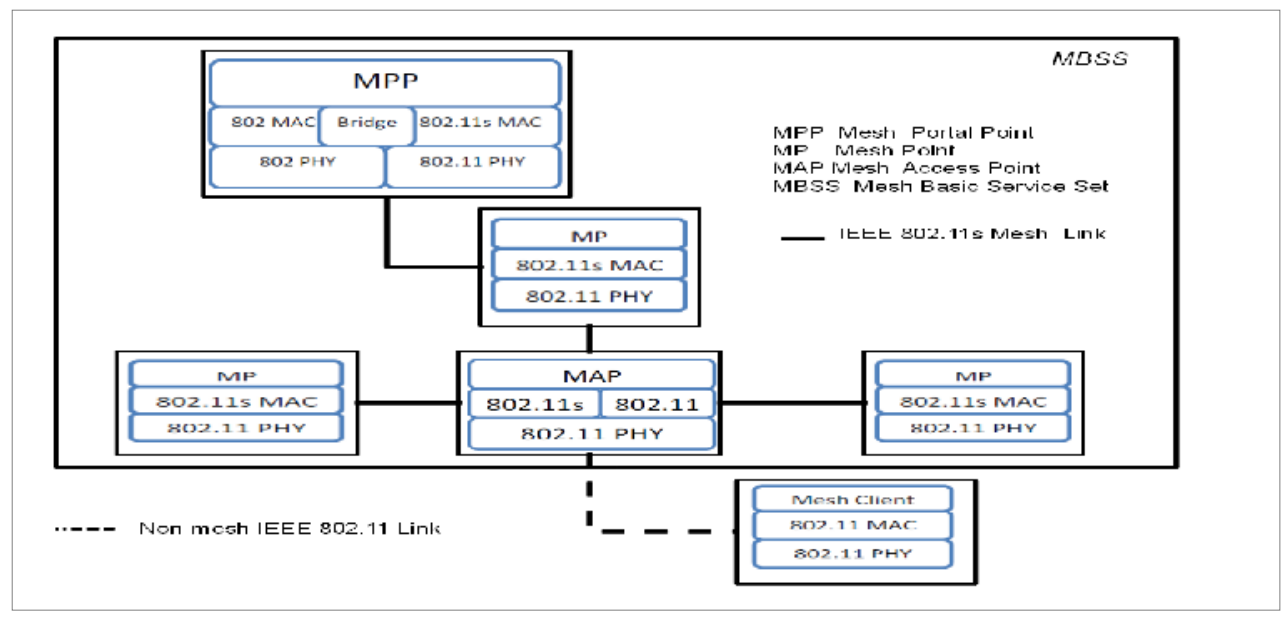

Figure 1. IEEE 802.11s WMN Architecture

\section{RELATED WORKS}

Some routing metrics specifically designed for WMN already exist in literature. In this section, we present ETX, ETT, WCETT and Airtime metrics. The comprehensive surveys of WMN routing metrics can be found in $[5,6,16]$. In order to improve the capacity of wireless mesh networks, each node in a network is equipped with multiple radio interfaces to assign multiple channels that help increasing the throughput of network[6].

\subsection{Expected transmission count (ETX)}

The Expected transmission count (ETX) [7] is one of the first routing metrics designed for wireless mesh networks(WMN). This is a link metric that estimates the number of transmission attempts (including retransmissions) required for a successful transmission on a particular wireless link. The ETX of a link is defined by (1) and the weight of a path is determined by summation of ETX values of all links along the path. ETX can be calculated as: 


$$
E T X=1 /\left(d_{f} \cdot d_{r}\right)
$$

Where $\mathrm{d} f$ and $\mathrm{d} r$ denote the delivery ratio in the forward and reverse directions, respectively. The ETX does not consider the impact of varying transmission rates of different wireless links and sizes of data packets [8]. ETX also does not consider the impact of intra-flow and inter-flow interference.

\subsection{Expected Transmission Time (ETT)}

The expected transmission time (ETT) metric calculates the time required to transmit a packet of size $\mathrm{S}$ on a link with a data rate B using (2). The ETT of a link is the duration of time a node uses the medium to successfully deliver a packet to the next hop. The ETT of the i-th link is defined by:

$$
E T T_{i}=E T X_{i} \cdot S / B_{i}
$$

Where :

- Bi : the data rate of the $\mathrm{i}$-th link.

- $\mathrm{S}$ : the packet size.

ETT and ETX not considers the presence of multiple channels. To resolve this problem WCET- T has been proposed in literature.

\subsection{Weighted cumulative expected transmission time (WCETT)}

The Weighted Cumulative Expected Transmission Time (WCETT) as a path metric for routing in multi-radio multi-channel WMNs[16]. To find paths with less intra-flow interference and channel diversity, the authors in [9] proposed WCETT metric for a path $\mathrm{P}$ of $\mathrm{n}$ hops by :

$$
\text { WCETT }_{P}=(1-\beta) \cdot \sum E T T_{i}+\beta \cdot \max _{1 \leq j \leq k} X_{j}
$$

Where :

$-0 \leq \beta \leq 1$ :the tunable parameter,

- Xj: represents the sum of ETT for the hops that are in the channel $\mathrm{j}$,

- $\mathrm{k}$ : the number of orthogonal channels.

The WCETT metric is composed by two components:

- The first defines the end-to-end delay experienced in a particular path,

- And the second accounts for channel diversity along the path.

However, WCETT considers the interference between the distant nodes and does not consider the link quality. To consider this, Airtime metric has been proposed. 
International Journal of Computer Networks \& Communications (IJCNC) Vol.5, No.2, March 2013

\subsection{Airtime}

The Airtime metric $c_{a}$ is the default routing metric specified in the draft of IEEE 802.11s [2] by:

$$
c_{a}=\left[O+B_{t} / r\right] \cdot\left[1 /\left(1-e_{f}\right)\right]
$$

Where:

- O : The channel access overhead,

- Bt : test frame size in bytes,

- $\mathrm{r}$ : data rate in $\mathrm{Mb} / \mathrm{s}$,

- ef: measured test frame error rate.

This metric defines the amount of channel resources consumed by transmitting the frame over a particular link, The path metric is the sum of metric values of all links in the path.

To cope with the issues stated above, we propose the New Metric for HWMP (NMH) to provide best path between the source and the destination. Our method is detailed in the following section.

\section{Proposed SOLUTiON}

In this section we describe our proposition. In order to decrease end-to-end delay and increase throughput, the proposed solution aims to compute hop by hop the value of new metric implemented with HWMP protocol. This new metric uses two components: channel diversity and packet hop delay. This new metric named New Metric for HWMP Protocol (NMH) determines the best path between the source and the destination.

This solution is implemented by all router's nodes. The proposed solution is composed by two steps:

- NMH metric computation,

- NMH implementation with HWMP.

These steps will be detailed in the next subsections. However the first subsection, presents HWMP protocol.

\subsection{MAC-layer Routing Protocol}

HWMP is a default MAC-layer protocol proposed by IEEE 802.11s draft [2]. It combines two modes: on demand reactive mode based on AODV [11], and tree-based proactive mode [11]. First mode is used to construct a path between MPs, while in second mode, a tree-based topology is created once an MP is configured as root. The two modes may be operated simultaneously, in this case, the mode that provides a best path is used. The HWMP uses Airtime Link metric. To communicate in the on-demand path discovery mode, the source MP include the destination MP address in PREQ (Path REQuest) frame and broadcasts it. Each intermediate node receives a 
International Journal of Computer Networks \& Communications (IJCNC) Vol.5, No.2, March 2013

PREQ, it verifies if it knows a path to destination, if it is the case, this node sends a PREP (Path REPly) frame back to source. When source can set a DO (Destination Only) flag in the PREQ frame, only destination node is allowed to respond with PREP frame. When destination node receives a PREQ, it unicast PREP in reverse path to the source node.

The proactive tree-based mode, proposed two mechanisms: Proactive PREQ and Proactive RANN (Root Announcement). In the first, when configured to work as a root MP, a node broadcasts a PREQ frame. This PREQ is sent periodically and every receiving MP broadcast the PREQ, which reaches all nodes in the MBSS. A node may sent a PREP frame back if it has data to send to the root node. The proactive RANN mechanism, instead of sending PREQs out of the root node, can flood the MBSS with RANN frames. To form a path to the root MP, nodes send a PREQ frame to the root MP, the root node responds each PREQ with PREP, thus forming a forward path from each MP to the root MP.

\subsection{NMH Metric Computation}

To determine the best path between the source and the destination, NMH defines a new metric for HWMP, namely NMH. Each intermediate node finds the maximum $\mathrm{Xj}$ of the two hops along with its precursors, when WCETT uses all the links of path operating in the channel $\mathrm{j}$.

To define a metric NMH, we combine WCETT with hop delay. Let $\boldsymbol{P}$ a path between the source node $\boldsymbol{S}$ and the destination node $\boldsymbol{D}$ :

$$
P=\left\{S, N_{1,} N_{2}, \ldots, N_{m}, D\right\} \text { and }\left\{N_{1}, \ldots, N_{m}\right\} \text { the set of intermediate nodes between } \boldsymbol{S}
$$

and $\boldsymbol{D}$.

We define $l_{i}$ the link between the nodes $N_{i-1}, N_{i}$ by:

$l_{i}=\left[N_{i-1}, N_{i}\right]$

And:

$l_{1}=\left[S, N_{1}\right]$
$l_{D}=\left[N_{m}, D\right]$

The metric value of link $\quad l_{i}$ is defined by:

$$
N M H_{l_{i}}=(1-\beta) \cdot E T T_{l_{i}}+\beta \cdot\left(D L_{i}-\max _{k} X_{j}\right)
$$


Where :

- DLi: The hop delay of link $l_{i}$.

- $\mathrm{Xj}$ : The two hop maximum metric value of the links in a path $\mathrm{P}$ operating on channel $\mathrm{j}$.

The NMH metric value of $\mathrm{P}$ is defined by following:

$$
N M H_{P}=N M H_{l_{l}}+\sum N M H_{l_{i}}+N M H_{l_{D}}
$$

The following subsection presents how HWMP uses NMH metric.

\subsection{NMH Implementation with HWMP}

In this subsection, we focus on MAC-layer routing protocol HWMP and NMH metric. HWMP uses NMH metric to find the best path between the source and the destination. Alongside the path

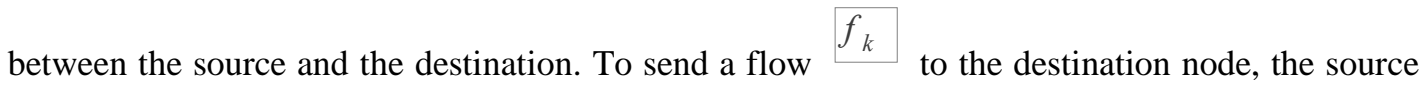
node initiate the Path Request (PREQ) frame with hop delay informations and broadcast it. Each intermediate node in MBSS increments NMH metric value and forward PREQ. After receiving PREQ, the destination node sends Path REPly (PREP) to the source node. Each intermediate node that receive PREP, increments metric value and forward PREP to the source node. After receiving PREP the source node transmits the flow $f_{k}$ to the destination node.

The following algorithm illustrates the metric updates . 
International Journal of Computer Networks \& Communications (IJCNC) Vol.5, No.2, March 2013

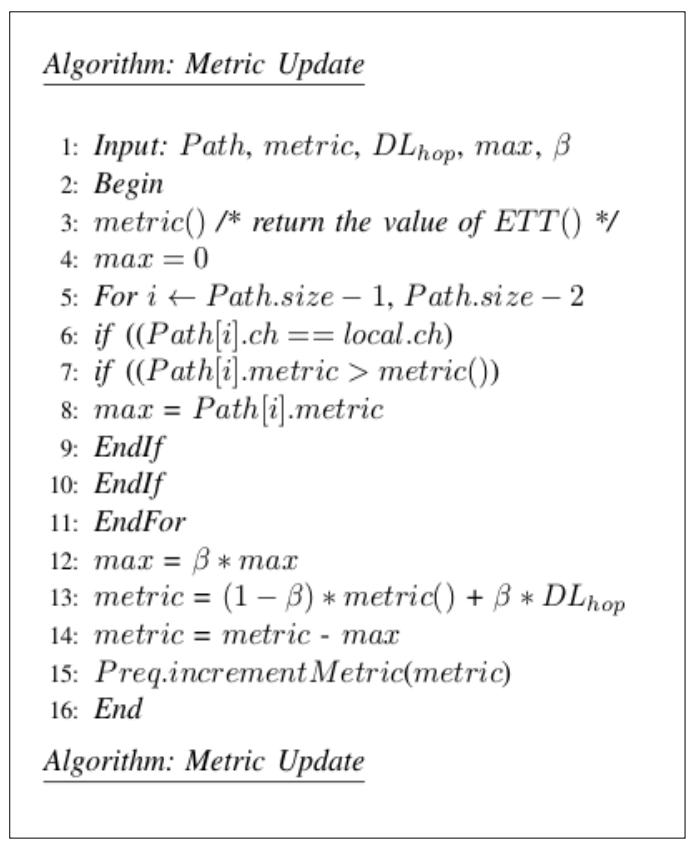

\section{SimUlation AND RESULTS}

We use NS3 [14] simulator to evaluate the performance of our proposed solution. The simulation compares WCETT and NMH implementations with the routing protocol HWMP. This simulation using two parameters: Throughput, End-to-end delay and two scenarios are used: first based on network size and second based on number of flows. The simulation parameters are presented in the Table 1.

\begin{tabular}{|l|l|}
\hline Parameter & Value \\
\hline Packet size & 512 bytes \\
\hline Data rate & $500 \mathrm{~kb} / \mathrm{s}$ \\
\hline$\beta$ & 0.5 \\
\hline Link capacity & $54 \mathrm{Mb} / \mathrm{s}$ \\
\hline Number of interfaces & 3 \\
\hline Flows number of scenario 1 & 4 \\
\hline Flows number of scenario 2 & $2-8$ \\
\hline Network size of scenario 1 & $20-55$ \\
\hline Network size of scenario 2 & 30 \\
\hline
\end{tabular}




\subsection{First scenario: based on network size}

In this scenario, we have assumed that 4 CBR flows exists in the grid topology mesh network.

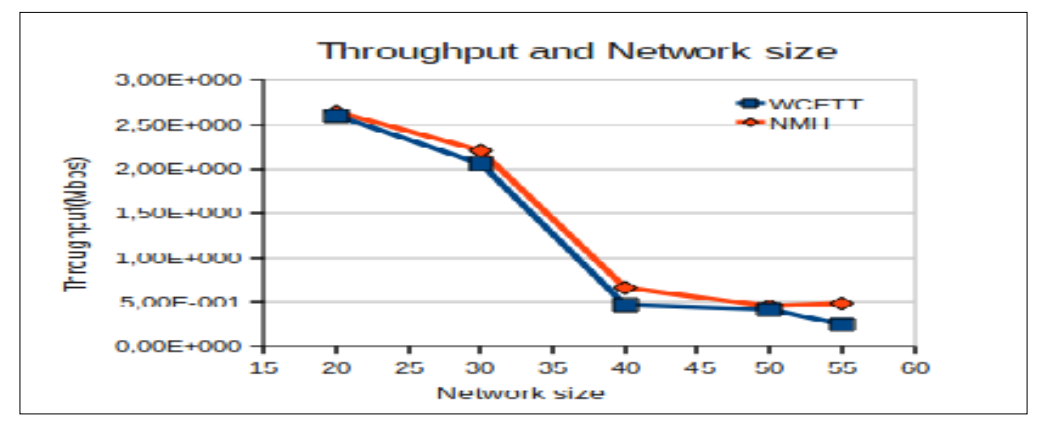

Figure 2. Throughput and network size

The network size increase from 20 to 55 nodes and the nodes are operated in multi-radio multi-

channel mode. The average results are plotted in the graphs Figure 2, Figure 3.

The average throughput achieved using NMH increased by $14.52 \%$ more than WCETT average throughput. Figure 2 shows the network throughput results.

When considering the end-to-end delay, NMH decreases it by $25.98 \%$ and provides a better average end-to-end delay than WCETT, Figure 3 illustrates this result.

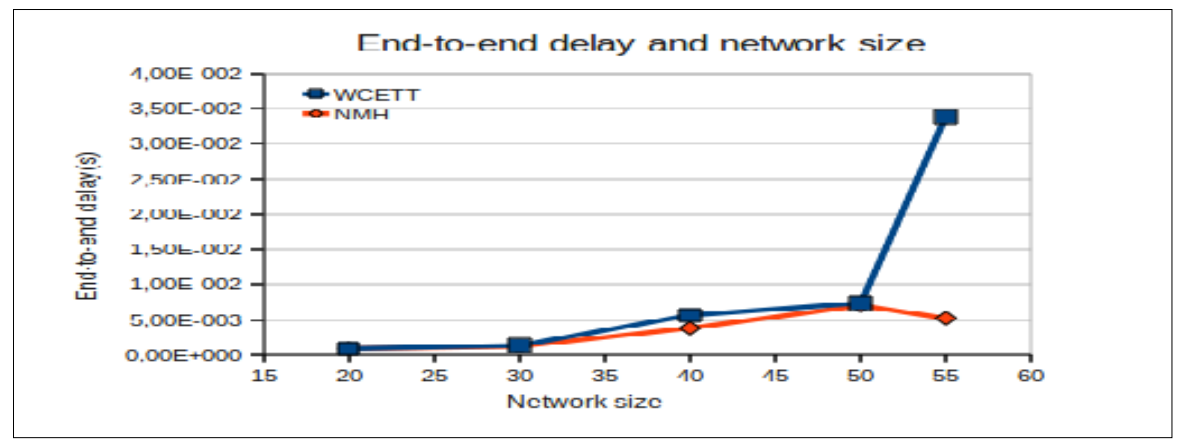

Figure 3. End-to-end and network size

\subsection{Second scenario: based on number of flows}

In this scenario, we have assumed that the network size is fixed in 30 nodes. The CBR flows increase from 2 to 8 and the nodes are operated in multi-radio multi-channel mode. The average results are plotted in the graphs Figure 4, Figure 5.

The average throughput achieved using NMH increased by $16.28 \%$ more than WCETT average throughput. Figure 4 shows the network throughput results. 


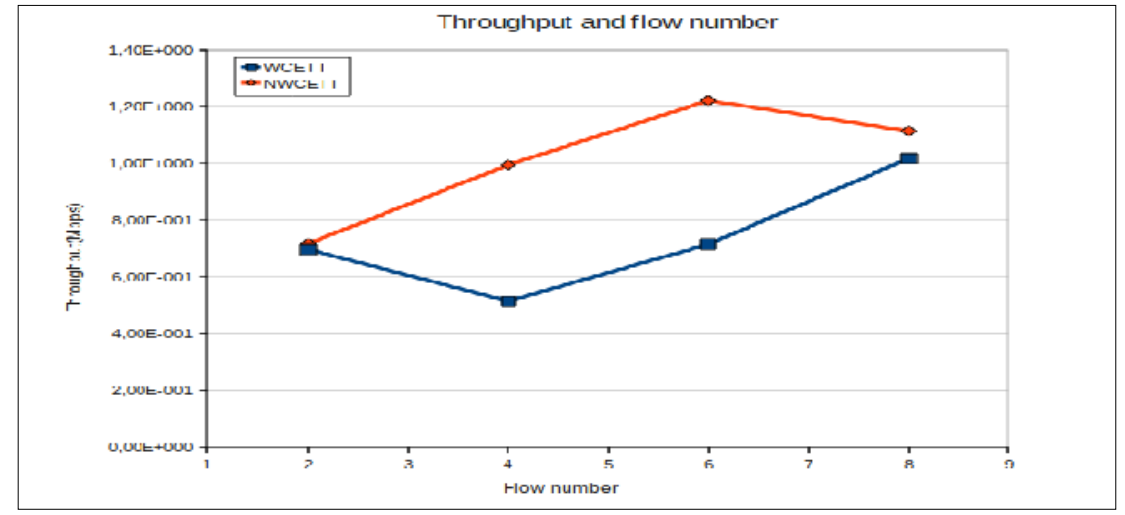

Figure 4. Throughput and flow number

When considering the end-to-end delay, NMH decreases it by $30.97 \%$ and provides a better average end-to-end delay than WCETT, Figure 5 illustrates this result.

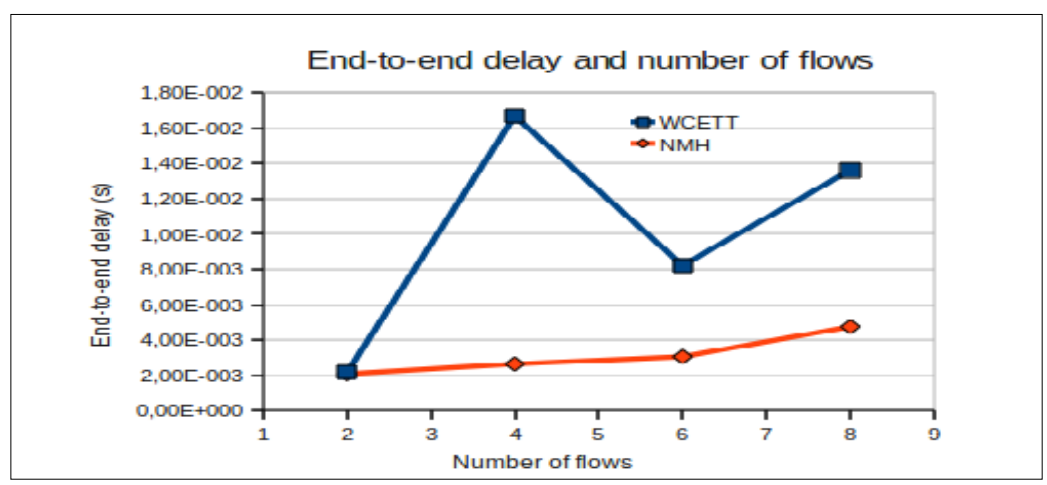

Figure 5. End-to-end delay and Number of flows

\section{CONCLUSION}

In this article, we have proposed a new metric based on two hop channel diversity and hop delay to increase a throughput and decrease end-to-end delay. The new metric is implemented by HWMP and operated in Multi-radio multi-channel mode based on $802.11 \mathrm{~s}$. We have shown the difference between the new metric NMH and the implementation of WCETT with MAC-layer routing protocol HWMP. Simulation results show that NMH performances compared with WCETT : decreasing end-to-end delay and increasing the network throughput. 
International Journal of Computer Networks \& Communications (IJCNC) Vol.5, No.2, March 2013

\section{REFERENCES}

[1] IEEE standard for Information technology - Telecommunications and informations exchange between systems - local and metropolitan area networks - Specific requirements - Part 11: Wireless Medium Access Control (MAC) and Physical layer (PHY) Specifications, IEEE Standard, Rev.802.11-2007, Jun.2007, sponsered by the LAN/MAN Standards committee of IEEE computer Society.

[2] IEEE 802.11s Task group, Draft Amendment to Standard for Information Technology Telecommunication and InformationExchange Between Systems - LAN/MAN Specific Requirements - Part 11: Wireless Medium Access Control (MAC) and physical layer (PHY) specification: Amendment: ESS Mesh Networking, IEEE P802.11s/D4.0, December 2009.

[3] A.Iera, A.Molinaro, S.Y.Paratore, G.Ruggeri, A.Zurzolo, "Making a mesh router/getway from a smartphone: is that a pratical solution?", Elsevier Ad hoc Netw.(2011),doi:10.1016/j.adhoc.2011.04.004.

[4] Conti, M., et al., Channel Assignment Strategies for Wireless Mesh Networks, in Wireless Mesh Networks,E. Hossain and K.Leung, Editors. 2007, Springer US. p.113-142.

[5] Si, W., S. Selvakennedy, and A.Y. Zomaya, "An overview of channel assignment methods for multi-radio multi- channel wireless mesh networks". Journal of Parallel and Distributed Computing, 2010. 70(5): p. 505-524.

[6] Tran Minh, T., G. Hong Yong, and S. Park Jeong. "A load aware hybrid channel assignment for Multi-radio Wireless Mesh Network". in Optical Internet (COIN), 2010 9th International Conference on. 2010.

[7] A.Alzubir, K.Babakar, A.Yousif, A.Aboubieda. "State of the Art, channel Assignment MultiRadio Multi-channel in Wireless Mesh Network". International Journal of computer Applications (0975-8887) Volume 37-No.4,January 2012.

[8] Rahman.M, A.Agarwal,and A.Alsarahn. "Capacity based channel assignment in multi-interface wireless mesh networks". 2008: IEEE.

[9] Skalli, H., et al.,"Channel assignment strategies for multiradio wireless mesh networks: issues and solutions". Communications Magazine, IEEE, 2007. 45(11): p. 86-95.

[10] Michelle X.Gong, Scott F.Midkiff, shiwen Mao,"On demande routing and channel assignment in milti-channel mobile ad hoc networks". Ad Hoc Networks 7(2009)63-78.

[11] C.Parkins, E.Belding-Royer,S.Das, "Ad Hoc on-demand-distance Vector(AODV) routing", IETF RFC 3561, 2003.

[12] Yang Y, Wang J, Kravets R (2006) Load-balanced routing for mesh networks. SIGMOBILE Mob Comput Commun Rev10(4):3-3.

[13] Holland G, Vaidya N, Bahl P (2001) A rate-adaptive mac protocol for multi-hop wireless networks. In: MobiCom 01: proceedings of the 7th annual international conference on mobile computing and networking. ACM, New York, pp 236-251.

[14] "NS-3", Network Simulator. Available: http://www.nsnam.org.

[15] Takeshi Ikenaga, Koji Tsubouchi1, Daiki Nobayashi1, Yutaka Fukuda, "Disjoint path routing fro multi-channel multi-interface wireless mesh network".(IJCNC) Vol.3, No.2, March 2011

[16] Md. Shariful Islam Muhammad Mahbub Alam Md.Abdul Hamid Choong Seon Hong. Sungwon Lee. "EFT: a high throughput routing metric for IEEE 802.11s wireless mesh networks ". Ann. Telecommun. (2010) 65:247-262. 